NADA BULIĆ

UDC: 801.73

Università degli Studi di Zadar

nbulic@unizd.hr

ANTE MATAN

Università degli Studi Juraj Dobrila di Pola

ante.matan@unipu.hr

\title{
LA VERSIFICAZIONE DI PASCHALIS VERRONVS NELLA VITA SANCTAE EUPHEMIAE
}

Sintesi. - L'articolo esamina i tratti versificativi del poemetto agiografico Vita diuae Euphemiae uirginis et martyris composto in 1232 esametri da Paschalis Verronus, Arcidiacono di Montepeloso, l'odierna Irsina nell'Italia meridionale, pubblicato nel 1592 a Napoli. La parte introduttiva del documento fornisce informazioni sulla vita e l'opera dello scrittore, nonché sul poemetto stesso, e nella parte centrale viene esplorata la sua tecnica di versificazione. Sulla base dell'analisi delle caratteristiche delle metriche interne ed esterne e dell'elaborazione statistica dei dati ottenuti dall'analisi, viene considerata la relazione tra Verronus e poeti classici come suoi modelli di ruolo in termini di versificazione. L'articolo osserva le caratteristiche della prosodia, la presenza e il rapporto di dattili e spondei, la presenza di cesure e dieresi, nonché la frequenza delle singole forme di esametri. Secondo questi criteri, si è scoperto che Paschalis Verronus era ben versato non solo negli autori classici, ma anche in quelli postclassici e cristiani. Virgilio in particolare ha lasciato su di lui un'influenza chiara e visibile, sia letteraria che versificativa. La ricerca ha inoltre dimostrato che Verronus costruì il suo poemetto combinando alternativamente schemi noti e frequenti, con quelli meno noti, cioè i suoi, il che potrebbe spiegare perché questa breve epopea agiografica, nonostante lo sforzo investito in essa dall'aspetto versificativo, è in certe sue parti incoerente, e il suo valore letterario, con tutta l'ampiezza dello spettro delle conoscenze e dei dati agiografici forniti dall'autore, è notevolmente diminuito. Gli autori concludono quindi che la familiarità dello scrittore con le soluzioni versificative, non solo principali ma anche meno conosciute, parla a favore di Verronus, che compone la sua epopea più come professionista che come poeta. E il valore dell'opera può essere trovato in un equilibrio di modelli di ruolo, da quelli generalmente noti, a quelli rari ed a quelli originari, malgrado che l'autore, in alcune parti, non riuscì a produrre un'opera di maggiore valore letterario-artistico.

Parole chiave. - versificazione, esametro, tipo di verso, Pasquale Verrono, agiografia.

Key words. - versification, hexameter, verse-type search, Paschalis Verronus, hagiography.

\section{Introduzione}

Alla fine del Cinquecento esce a Napoli, dall'officina di Orazio Salviani e dalla tipografia di Giovanni Giacomo Carlin e Antonio Pace, un libretto intitolato Vita diuae Euphemiae uirginis et martyris. L'autore di questo poemetto agiografico è Pasquale Verrono (Paschalis Verro- 
nus), che all'epoca della pubblicazione, nel 1592, era l'arcidiacono di Montepeloso, l'attuale Irsina, un luogo dell'Italia meridionale, nella regione della Basilicata (antica Lucania), provincia di Matera. L'opera era del tutto sconosciuta già verso la metà del Settecento ${ }^{1}$ ed è stata riscoperta appena negli anni ' 80 dello scorso secolo, con uno studio storico-letterario e una traduzione in italiano pubblicata da Don Nicolino Di Pasquale ${ }^{2}$. In occasione del $1700^{\circ}$ anniversario dell'arrivo a Rovigno del sarcofago con il corpo della Santa, nel 2004 è stata pubblicata la prima e finora unica traduzione in lingua croata. ${ }^{3}$

È noto che il suo modello letterario non è necessariamente anche un modello di versificazione, cioè non si può parlare, in maniera fondata, "dell'eleganza o fluidità e dell'onda armoniosa del verso virgiliano", senza che ciò sia metodologicamente supportato dai dati ottenuti mediante una rigorosa elaborazione statistica. Solo tale ricerca ci darà una risposta precisa alla questione inerente i modelli di versificazione adottati da Pasquale Verrono nella costruzione del proprio esametro. Le statistiche porteranno così alla luce alcuni dati inattesi e forniranno un confronto tra l'esametro di Verrono ed i modelli classici.

L'esimio professor Branimir Glavičić ${ }^{5}$ ha migliorato e innalzato ad un livello superiore i principi posti da Duckworth ${ }^{6}$, insistendo sui dati ottenuti dallo studio della metrica interna. La ricerca qui condotta è basata sui principi metodologici di Glavičić, ma è stata ampliata mediante l'utilizzo delle moderne tecnologie che recentemente ci sono state messe a disposizione. ${ }^{7}$

\section{Paschalis Verronus}

Questo è uno di quegli esempi evidenti della nota locuzione exegi monumentum, aere perennius, poiché l'autore sarebbe rimasto del tutto sconosciuto, se non fosse stato per la Vita di Sant'Eufemia. I dati sull'autore sono alquanto scarsi. Ianora sostiene che egli fosse stato dottore in entrambi i diritti, arcidiacono della cattedrale di Montepeloso e "felicissimo poeta" 8 , che nel 1592 scrisse "un bel poemetto latino". Dagli scritti capitolari si ricava inoltre che Verronus "cantò

${ }^{1}$ Ianora, 1901: 544.

${ }^{2}$ Di Pasquale, 1987.

${ }^{3}$ Matan, 2004.

${ }^{4}$ Di Pasquale, 2001: 44.

${ }^{5}$ Glavičić, 2001.

${ }^{6}$ Duckworth, 1969.

${ }^{7}$ L'analisi dei versi secondo il metodo di Glavičić è stata fatta diversi anni fa, ma non c'era tempo per una sintesi dei dati. Purtroppo è stato solo nell'autunno del 2018 che ho preso conoscenza delle possibilità offerte da http://www.pedecerto.eu/public/index, quindi valeva la pena controllare i vecchi dati, dove si è creato un momento completamente nuovo nelle possibilità di analisi della metrica interna, che a sua volta ha portato dovendo iniziare a rianalizzare.

${ }^{8}$ Ianora, 1901: 582. 
l'epistola" il 21 maggio 1567, fu ordinato diacono il 17 febbraio 1570 e sacerdote il 23 dicembre 1570; come cappellano divenne membro del capitolo il 19 novembre 1574 e il 13 agosto 1577 fu eletto canonico, mentre morì nel $1595 .^{9}$

Di Pasquale afferma che Verronus nacque a Montepeloso intorno al 1545, da un'importante famiglia che nel Cinquecento ebbe anche altri membri che si distinsero "nella carriera degli studi". Fu un sacerdote diocesano, poi arcidiacono del capitolo e molto devoto a Dio. ${ }^{10}$ Può considerarsi il primo autore di Montepeloso la cui opera è stata completamente conservata, dato che ci è pervenuto un solo epigramma dal titolo Ad invictissimum regem Hispaniarum, scritto dal vescovo Leonardo De Carminis (vescovo di Montepeloso 1491-1499). ${ }^{11}$

Il nome italiano del nostro autore è quello di Pasquale Verrone, ${ }^{12}$ in linea con la variante latina che compare sulla prima pagina dell'edizione: “( ...) per reverendum D. Paschalem Verronem Archidiaconum Montis Pilosi". ${ }^{13}$ Poco più avanti troviamo invece: "Paschalis Verronus a Monte Piloso Archidiaconus", ${ }^{14}$ oppure: "Paschalis Verroni a Monte Piloso Archidiaconi". ${ }^{15}$ È possibile che nel caso di "Verronem" ci sia stato uno slittamento sotto l'influenza dell'italiano, ma ciò non esclude in alcun modo un'evidente esitazione nello scegliere la declinazione del nome latinizzato, e la risposta a questa domanda va cercata altrove. Pertanto, è stato citato in quest'articolo assecondando la versione latina di Paschalis Verronus.

\section{Vita diuae Euphemiae}

Dal 1750 al 1761 il vescovo di Montepeloso fu mons. Bartolomeo Coccoli, che nel 1755 scrisse, in italiano, la biografia di S. Eufemia, nel manoscritto - Vita di S. Eufemia ${ }^{16}$ che si basava in larga misura sull'agiografia di Verronus, di cui sono stati conservati solo 72 versi. ${ }^{17}$ Oggi possiamo stabilire che Coccoli aveva conservato $i$ seguenti versi: 920-926; 943-944; 957-975; 978-983; 1010-1024; 1055$1060 ; 1146-1162,{ }^{18}$ notando che secondo Coccoli i versi $1055-1060$ seguivano quello $1162 .{ }^{19}$ Ianora, che aveva dinanzi agli occhi i versi di Coccoli, mediante la trascrizione del Canonico Tommaso Torrio, rinuncia a ogni responsabilità per la correttezza dei versi da lui ripor-

\footnotetext{
${ }^{9}$ Ianora, 1901: 582.

${ }^{10}$ Di Pasquale, 2001: 41.

${ }^{11}$ Di Pasquale, 2001: 42.

12 Ianora, 1901: passim; Di Pasquale, 2001: passim.

${ }^{13}$ Verronus, 1592: 1.

14 Verronus, 1592: 2.

${ }^{15}$ Verronus, 1592: 3.

${ }^{16}$ Di Pasquale, 2001: 105.

${ }^{17}$ Ianora, 1901: 544.

${ }^{18}$ Cfr. Ianora, 1901: 541-544 e Verronus, 1592: 22-25; 27.

${ }^{19}$ Ianora, 1901: 544.
} 
tati, ${ }^{20}$ per cui non va biasimato per alcune piccole differenze: sciebatscibat (v. 920), Magna tutela, - Moenia, tutela, (v. 921), Patavique pergamena - Patavi quae pergama (v. 924), dixit - dictis (v. 959), dono fere - dona fero (v. 963), Migrantem - Nigrantem (v. 973), impulsu - impulsus (v. 974), In - Tu (v. 980), Mutantes - Nutantes (v. 1148), nemini - memini (v. 1159), mentre refet-feret (v. 1055) probabilmente mostra un lapsus typi. Certamente, queste differenze nei versi 920 , 924, 963 e 1159 rompono la struttura metrica e non sono esametri, mentre il v. 921 è accettabile in termini di criteri di metrica esterna, ma non di quella interna.

L'edizione, come già accennato, fu trovata da Di Pasquale, che essendo alquanto modesto descrive il fatto solo come: “...fortunato ritrovamento dell'intero poemetto latino...", ${ }^{21}$ e concepisce la propria edizione in modo tale (1a edizione 1987, 2a edizione (ristampa) 2001) che, assieme al facsimile del testo latino e alla traduzione in italiano (in prosa), ci presenta diversi dati storici contenuti nel poemetto, riferendosi a Calcedonia, Rovigno, al manoscritto di mons. Coccoli (con un estratto dal manoscritto), ai dati storici e alla diffusione del culto di S. Eufemia, completando il tutto con una breve raccolta di inni in onore alla Santa e con la preghiera di mons. Lupoli. Dal punto di vista letterario e metrico, il Verronus ha trattato anche la Vita in modo molto scarno e troppo generico. ${ }^{22}$

Di Pasquale descrive le opere di Verronus, ma anche quelle di De Carminis e Maranta, come scritti di valore "... non particolare, che però vanno ad arricchire la serie di poemetti, elegie, egloghe ed epigrammi che fiorivano in tanti paesi e città d'Italia nei quali spesso, all'eleganza del dire, non sempre si accompagnava lo slancio dell'ispirazione e la vigoria del sentimento." ${ }^{23}$ Tra i più eminenti umanisti della Basilicata indica Giovanni Albino e Gabriele Altilio, collocando la persona e l'opera del Verronus tra coloro che, "pur provenendo da comunità minori, seppero unire la fede cristiana e la cultura umanistica, dedicando un'ode alla santa patrona e ottenendo così, meritatamente, un posto d'onore nella letteratura rinascimentale." ${ }^{24} \mathrm{La}$ sua Vita, in assenza di altre opere, è sufficiente per qualificare il Verronus "come una voce valida di un figlio degno della sua patria, che ha trovato un senso nella propria vita, nella fede, nel servizio alla Chiesa e nello studio degli autori classici." 25

Lo studioso osserva quindi che il Verronus usò la mitologia con moderazione, in conformità con il carattere religioso dell'opera, nonostante "il poemetto difetti di originalità e manchi di una certa sobrietà

\footnotetext{
${ }^{20}$ Ianora, 1901: 544.

${ }^{21}$ Di Pasquale, 2001: 8.

${ }^{22}$ Di Pasquale, 2001: 41-45.

${ }^{23}$ Di Pasquale, 2001: 42.

${ }^{24}$ Di Pasquale, 2001: 43.

${ }^{25}$ Di Pasquale, 2001: 44.
} 
che lo avrebbe reso più agile, mentre a volte scade in ripetizioni artificiose ed inutili." 26

Riguardo alla versificazione, scrive così: "Al contrario è evidente l'eleganza del verso che denota nell'autore lo studio appassionato dei classici. Sono frequenti i ricordi di Virgilio, si coglie facilmente la fluidità e l'onda armoniosa del verso virgiliano proprio lì dove si nota la maggiore frequenza della cesura semiquinaria rispetto alla settenaria, la giusta proporzione metrica fra dattili e spondei, la prevalenza del verso spondaico, la frequente separazione del quarto piede dagli ultimi due." 27 Tuttavia, i risultati della nostra ricerca dimostreranno che non è proprio del tutto così.

Va poi a cogliere una certa ispirazione nel Verronus desunta dal De partu Virginis di Sannazzaro e La Cristiade di Gerolamo Vida, ovvero da due evocazioni dantesche, concludendo: "E così in tutta la narrazione non vengono mai meno i ricordi classici, l'eleganza e la sonorità del verso, l'abilità nel maneggiare la lingua che compensano un po' la stanchezza del lettore in determinati punti, anche se poi il poeta si riscatta nella bella e commovente preghiera finale che rivolge alla Santa quando Le affida la tutela del paese e delle sue alterne vicende." ${ }^{28}$

\section{La versificazione del Verronus}

\section{Prosodia}

La sua prosodia è perlopiù orientata classicamente, con allungamenti o abbreviamenti più o meno comuni, a seconda delle necessità, come: sŏpito (114), uāfer (157), credǒ (185), postulǒ (258), fāc (252, 265), orö 265, uōlunt (326) - ma uŏlunt (631), eódem (457), quōtus (1169), Deniqué (1202), dove l'autore ovviamente tendeva a una soluzione più rapida, cioè prediligeva l'influenza del cursus, perché era abbastanza esperto di prosodia e versificazione antica. Nei casi dove sono possibili i doppietti, li usa anche come tali: nemō 265, nemó 1119, sibi 1016, 1073, mentre negli altri casi assume sempre la forma di pirrichio; ciò vale sempre per ego, usato rigorosamente in modo classico. Negli esempi těrrendam (567) e rēsǐstit (986), laddove serviva oltreppassava i limiti, salvo che non si trattasse di prosodie medievali.

Nel caso del nome dell'arcangelo, non ha seguito l'anapesto di Venanzio Fortunato (carm. spur. 1, 185 e 317), ma lo pose come bacchio Müchāèl (279).

Troviamo nel testo un verso apparentemente ipermetrico, 1200: Fornacis, laquei, serrae, lapidumque, leonumque, / Immitis..., ma questo è risolto dalla sinalefe col verso seguente.

\footnotetext{
${ }^{26}$ Di Pasquale, 2001: 44.

${ }^{27}$ Di Pasquale, 2001: 44.

${ }^{28}$ Di Pasquale, 2001: 44-45.
} 
La desinenza del perfetto è regolarmente -ērunt, però si può trovare anche quella in -êre (41: Ingens est Asiae (sed quam dixere minorem)). L'allungamento di -que in arsi si trova una volta, però molto spesso la particola è soggetta a sinalefe. La sinizesi in aperuit (v. 702) mette in salvo la struttura del verso.

Tra le prosodie doppie bisogna sottolineare: virgó (vv. 37, 288, 431, 500, 707, 709, 797, 946, 1146), e virgō (v. 30*, 33, 232, 447, $\left.545^{*}, 581,640^{*}, 732,765,929^{*}, 950,957,1059,1190^{*}, 1221^{*}\right)$, utilizzate approssimativamente in un uguale numero d'istanze. ${ }^{29}$

Non sono stati riscontrati esempi di iato ne $-s$ caduca finale.

\section{Sinalefe}

La sinalefe si verifica in 247 casi, cioè in $20,04 \%$ dei versi, in 13 dei quali si tratta di doppia sinalefe e in un verso di tripla sinalefe, che è anche il massimo che Verronus usa. Nel caso della doppia sinalefe, si vede che è distribuita uniformemente, di solito a sinistra e a destra della cesura centrale ${ }^{30}$, cosa che fa principalmente il nostro autore.

17: In Christi_Athletas; quae contra_his parta trophaea ${ }^{31}$, dove la sinalefe impedisce l'inconveniente prima dieresi (D), ma anche la cesura semiseptenaria (H), lasciando spazio alla regolare bucolica (B); con la sequenza quae contra his ha ottenuto la terza dieresi (3D), quale poteva essere evitata rispettando la legge di Marx, che risulterebbe con la $\mathrm{H}$ invece di 3D: *contra_his quae.

91: Exosa $\_$a teneris, cupiensque $\_$a munere turpi ${ }^{32}$, impedita l'inconveniente $\mathrm{D}$, ma anche la $\mathrm{H}$.

100: Auersi_a sacris, dirique_a Praesidis ira. ${ }^{33}$, come nel verso precedente.

164: Eia_age, neglectos una_instauretis honores, impedita l'inconveniente 3D, ma anche la cesura dopo il primo trocheo (1TR), però riscontriamo la $\mathrm{D}$.

714: Assensere_omnes, nugisque_his denique Priscus ${ }^{34}$; dove la prima sinalefe impedisce ogni cesura fino alla semiquinaria (P),

29 L'asterisco indica gli esempi in cui la parola si trova nell'ultimo piede dell'esametro. Sebbene l'ultima sillaba sia generalmente considerata in teoria come una anceps, qui seguiamo la regola che alla fine del verso c'è una pausa che allunga la sillaba precedente, di conseguenza il secondo emistichio è fisso, tranne nel caso di un verso spondiaco, v. Glavičić, 2001.

${ }^{30}$ Cfr. Verg. Aen. 9, 595; Glavičić, 2001: 28.

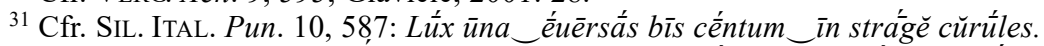

${ }^{32}$ Cfr. STAT. Theb. 6, 752: Ármōrum í̀n spécŭlís, ădĭtúsque ād uúlnĕră cláusi.

${ }^{33}$ Cfr. STAT. silu. 5, 3, 269: Témptāntem ét uīuós mōlìtum ìn Tártără gréssus;

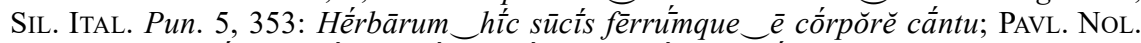

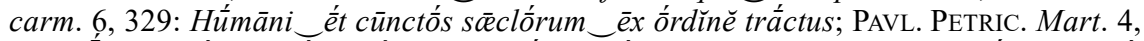

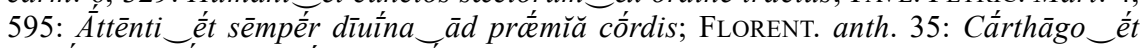
dūlcís, Cārthágo_èt néctărě suáuis. 
mentre l'altra impedisce la cesure dopo il terzo trocheo (KT) e la H, ma avendo la B.

800: Vt morbo_affecti, uitaque_extrema ferentes: ${ }^{35}$; dove l'incisione affianco alla prima arsi (J), con la P sintatticamente segnata, la seconda sinalefe impedisce cesure fino all'incisione affianco al $5^{\circ}$ trocheo (5TR).

840: Inque Deum_inque_homines exactae crimina uitae: ${ }^{36}$, aparte l'inconveniente $\mathrm{D}$, è impedita anche la semiternaria $(\mathrm{T})$.

845: Quaeque erat in terris humilis, nunc maxima coelo_est ${ }^{37}$; la sinalefe produce la $\mathrm{D}$ e la struttura stessa del verso è assai frammentata $(\mathrm{D}+\mathrm{T}+\mathrm{P}+\mathrm{H}+\mathrm{B} / 5 \mathrm{D})$.

867: Postquam_expleta sitis confossa_in Virgine Prisci ${ }^{38}$; le sinalefi producono la più rara incisione affianco al $2^{\circ}$ trocheo (2TR), cioè la $\mathrm{B}$, accompagniate dalla $\mathrm{P}$ non sintatticamente segnata.

937: Fluminis exigui, ¿hic summa ad fastigia surgit; dove al posto della $\mathrm{T}$ dell' antico modello $\mathrm{T}+\mathrm{KT}+\mathrm{H}$, viene la $\mathrm{D}$.

952: In monte_imprimis humano_est lumine uisus; impedite la $\mathrm{T}$ e la $\mathrm{H}$, che risulta con la $\mathrm{J}$ e la $\mathrm{B}$, mentre la cesura centrale non è sintatticamente segnata.

955: In monte _is moritur, de monte_ad sidera coeli; la sinalefe impedisce la D, altrettanto la $\mathrm{H}$, come la regolare B.

Verronus ha solo una eccezione, ponendo due sinalefi nella seconda parte dell'esametro, impedendo la H, 389: Praesidis ad sedes, prauique_ante_ora Tyranni:; senza pausa dalla $\mathrm{P}$ al quinto trocheo (5TR).

Nell'esempio della triplice sinalefe, 199: Supplicia,_atque_amens animum circum_omnia uertit:, due sinalefi impediscono ogni incisione fino alla $\mathrm{P}$, cioè l'inconveniente $\mathrm{D}$ e regolare $\mathrm{T}$, mentre la terza impedisce la regolare $\mathrm{B}$.

La sinalefe impedisce la $\mathrm{H}$ in $7,19 \%$ di casi, mentre nel caso della P si tratta di appena sette esempi $(0,67 \%)$ :

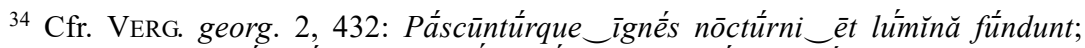

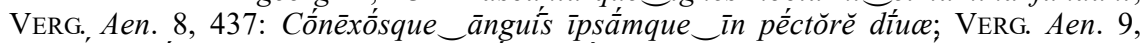

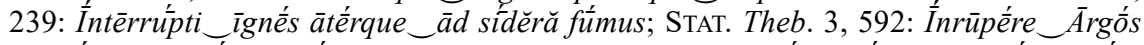

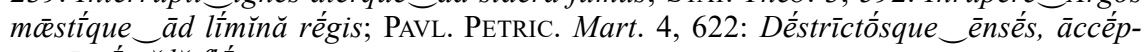
to $\_\bar{e} t$ túrgl̆dă flấtu.

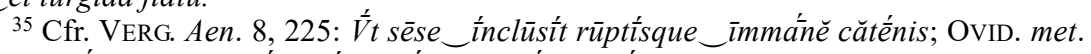

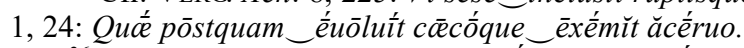

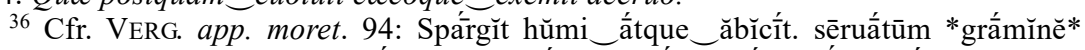

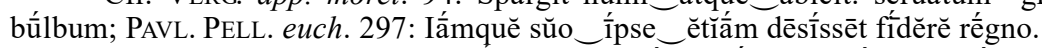

${ }_{37}$ Cfr. Ovid. am. 1, 12, 27: Êrgo ěgŏ uốs rēbú́s dǔplǐcếs prō nốmǐně sếnsi; LvCAN. Phars. 9, 511: Vếntum ěrăt ắd tēmplúm, Lỉby̌cís quōd gếntǐbŭs únum; ProBA cento 422: ńlum ěgŏ pếr flāmmás, ăgěrếm sī Sýrtỉbŭs éxul; ANTH. Lat. 493a, 5: V́nde_ěgó tế, lēctốr, rělěgî́s qui_hǣc sếdŭlŭs, ốro.

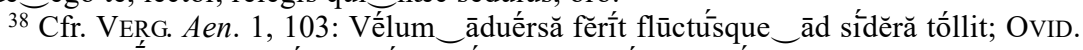
met. 2, 603: Árma àdsuéttă căpìt flēxútmque à cốrnùbŭs árcum; VAL. FL. Argon. 1,

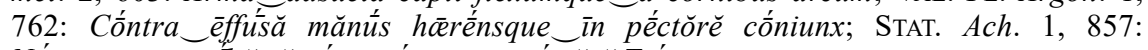
Nứsquam_ōccultŭs ămó̀r, tōtốque in péctŏrĕ Trốiast. 
281: Docta queat superare, Chumiles ingressus in aedes, ${ }^{39}$;

405: Dente rotas, roseisque humeris conspersa cruore;

430: Terribiles non ira_oculos, non uerba minantis ${ }^{40}$;

592: Incolumis, ferrique_acies obtusa repente;

906: Munus obit, rerumque_habiles sibi ducit habenas: ${ }^{41}$;

1176: Labentes fulcite_animas, populosque docete;

1230: Vulneribus; quae cladem animis mortemque minantur; cosa da Verronus risolta con il modello $\mathrm{T}+\mathrm{H}$, dove una delle cesure può essere anche sintatticamente segnata, e il modello ampiato dall'incisione dopo il primo trocheo (1TR) (v. 281, 405, 906), o seconda dieresi (2D) (v. 430, 1230).

Non si riscontra la sinalefe in parole monosillabiche, eccetto con il verbo esse, come ad esempio 163: Quaeue boni tanti uestra_est iactura? quis auctor?, 408: Haec Regi Dominoque suo sic ore loquuta_est, 590: Suppliciter, Dominoque suo sic ore loquuta_est, 845: Quaeque erat in terris humilis, nunc maxima coelo est, ${ }^{42}$ te 952: In monte imprimis humano_est lumine uisus.

Il nostro autore quindi differisce in percentuale di sillaba ammissibile da Virgilio (41,98\%), sebbene abbastanza vicino nelle eclog. $(21,59 \%)$, mentre i valori più simili si trovano con CYPR. GALL. $(20,02 \%)$, ANTHOL. LAT. (19,69\%) e AVIEN. (19,72\%), ILIAS LATINA $(21,51 \%)$, PROP. (21,64\%), HOR. (21,68\%), GERM. (21,97\%), PAVL. Nol. $(22,12 \%)$, da cui non differisce tanto STAT. nelle silu. $(22,58 \%$, totale però $29,89 \%$ ). Sotto questo aspetto Verronus, eccezione fatta per le eclog., è più vicino a Ennio $(15,96 \%)$ che a Virgilio.

\section{Emistichio anteriore}

Quando si tratta di dattili e spondei, cioè la loro frequenza, i numeri stanno da Verronus come segue. Il tipo più frequente è il DSSS, al secondo posto si trovano DDSS e DSDS, mentre al quarto il tipo DSSD. Segue il SDSS e al sesto posto DDDS e DDSD, mentre all'ottavo posto riscontriamo SSDS. Dal $9^{\circ}$ al $12^{\circ}$ posto seguono DSDD, SSSS, SDDS e SDSD. Il rapporto di dattili e spondei nei quattro tipi più frequenti risulta $7: 9$, nei primi otto $15: 17$, e nei primi dodici $22: 26$.

${ }^{39}$ OvID. met. 8, 584: Tántŭs ĕrá̀m părĭtérque_ănĭmî́s ìnmánŭs ĕt úndis; PAVL. NOL.

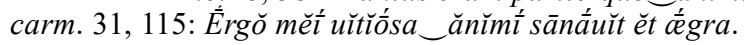

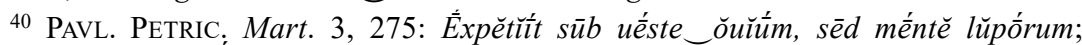
CORIPP. Ioh. 2, 264: İncìpiúnt èt túta ăciés pēr cástră rĕcóndunt.

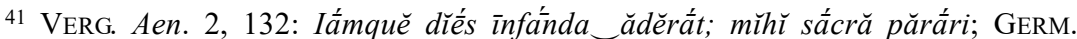
Arat. 399: Mứltă dĕdî́t nātúra_hŏmìnt rătă sígnă sălứtis; STAT. Theb. 11, 370: Tứrbă rŏgấnt āmbóeque_ăcǐés; rŏgăt íllă sướrum.

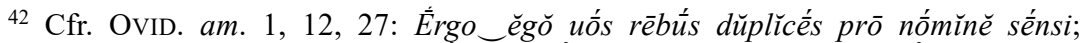

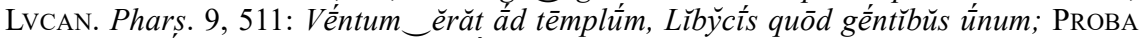

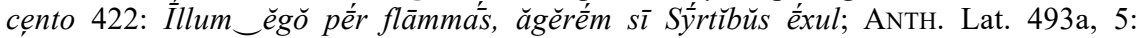

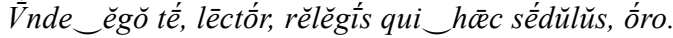




\begin{tabular}{|c|c|c|c|c|}
\hline & 음 & & $\widetilde{\widetilde{\Xi}}$ & ลे \\
\hline & 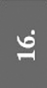 & 鏟 & $\Xi$ & $\stackrel{m}{\Rightarrow}$ \\
\hline & $\ddot{\theta}$ & 容 & I & $\stackrel{m}{-}$ \\
\hline & $\dot{I}$ & $\begin{array}{l}\text { 章 } \\
\frac{8}{2}\end{array}$ & ๙ิ & 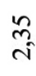 \\
\hline & $\ddot{g}$ & 䆚 & $m$ & io \\
\hline 0 & $\mathcal{N}$ & 密 & F & $\stackrel{?}{\text { f }}$ \\
\hline $\bar{z}$ & $\dot{\Xi}$ & 享 & $\approx$ & 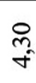 \\
\hline$\tilde{\Xi}$ & $\stackrel{\ominus}{ }$ & $\begin{array}{l}\text { v } \\
\text { as }\end{array}$ & 8 & $\begin{array}{l}\hat{\infty} \\
\underset{f}{f}\end{array}$ \\
\hline 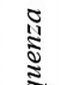 & $a^{\circ}$ & ถิ & $\widetilde{\sigma}$ & ô. \\
\hline & $\infty$ & 章 & $\mathscr{0}$ & $\begin{array}{l}\vec{n} \\
\text { in }\end{array}$ \\
\hline 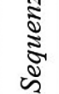 & ๑ & 命 & $\approx$ & $\stackrel{8}{0}$ \\
\hline 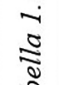 & ๑ & 合 & $\approx$ & $\stackrel{8}{0}$ \\
\hline & vi & 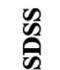 & $\because$ & $\frac{0}{6}$ \\
\hline & & $\begin{array}{l}\text { 悹 } \\
\text { 点 }\end{array}$ & $\vec{a}$ & $\stackrel{\infty}{m}$ \\
\hline & غ่ & 气̆ & $\underline{\overrightarrow{0}}$ & $\begin{array}{l}\stackrel{8}{0} \\
\text { m }\end{array}$ \\
\hline & r & 銅 & $\underline{0}$ & $\stackrel{\text { ¿ }}{\text { m. }}$ \\
\hline & & $\begin{array}{l}\text { va } \\
\text { b. }\end{array}$ & $\stackrel{n}{\sim}$ & $\begin{array}{l}\text { ñ } \\
\text { s }\end{array}$ \\
\hline & & $\stackrel{2}{\circ}$ & 远 & $\partial^{\circ}$ \\
\hline
\end{tabular}

In termini di rapporto tra dattili e spondei nelle otto forme più frequenti di esametri, il nostro autore coincide con la Anthol. Lat., a differenza di Virgilio, Cicerone, Catullo, Germanico, Manilio e Cipriano Gallo (tutti con 12 dattili e 20 spondei), nonché autori con un nu- 
mero più elevato di dattili (Ovid., STAT., AUSON., PAVL. NOL. e VEN. FORT.). Non si allontana tanto da Lucrezio e da Persio (14D/18s). In Virgilio non risultano le otto forme più frequenti né DSSD né DDSD (al $4^{\circ}$ e $7^{\circ}$ posto da Verronus), al $4^{\circ}$ si trova SDSS che da Verronus occupa il $5^{\circ}$, mentre lo stesso posto da Virgilio tiene la forma SSSS, che da Verronus si trova appena al $10^{\circ}$. Lo stesso vale per la forma SDDS che non trova posto nei primi otto posti da Verronus, però si trova da Virgilio all'ottavo. In entrambi il $6^{\circ}$ posto occupa la forma DDDS, mentre il SSDS ci si trova da Virg. al $7^{\circ}$ posto e da Verronus all'ottavo.

Tabella 2. Rapporto di dattili e spondei e frequenza di tipi

\begin{tabular}{|c|c|c|c|c|c|}
\hline \multicolumn{5}{|c|}{ Rapporto di dattili e spondei } \\
\hline \multicolumn{2}{|c|}{$1-4$} & \multicolumn{2}{c|}{$1-8$} & \multicolumn{2}{c|}{$1-12$} \\
\hline dattili & spondei & dattili & spondei & dattili & spondei \\
\hline 7 & 9 & 15 & 17 & 22 & 26 \\
\hline & & & & & \\
\hline
\end{tabular}

\begin{tabular}{|c|c|c|c|}
\hline \multicolumn{4}{|c|}{ Frequenza di tipi } \\
\hline 1 & $1-4$ & $1-8$ & $1-12$ \\
\hline $17,45 \%$ & $50,97 \%$ & $74,83 \%$ & $92,45 \%$ \\
\hline
\end{tabular}

La forma DSSD da Verronus è al $4^{\circ}$ posto, nella Anthol. Lat. appena $\mathrm{al} 7^{\circ}$, quindi le forme SDSS, DDDS e DDSD occupano un posto più elevato. Altre somiglianze e differenze possono essere viste nella Tabella $3 .{ }^{43}$

Seguendo l'esempio di Omero, è una buona pratica di versificazione mettere una parola pesante nell'emistichio anteriore, in modo che poggi con la sua estremità sulla cesura centrale, e a volte sulla seconda dieresi. ${ }^{44}$ Verronus colloca la parola difficile soprattutto nell'emistichio anteriore, ma non segue l'esempio di Omero. Infatti, nella maggior parte dei casi la colloca all'inizio dell'incisione dietro il secondo trocheo (2TR) (13 volte), come ad esempio v. 8: Penthesilea ruat: ...; 45: Diuitiisque potens. his ...; 51: Maximianus erat rebus, ...; oppure 1193: Insidiisque premunt: ...; e solo una volta all'inizio della $\mathrm{P}$, per lo più sintatticamente segnata, 44: Bizantinorum, muris .... No-

\footnotetext{
${ }^{43}$ I numeri sono calcolati in base alla tabella degli schemi metrici generali disponibile su http://www.pedecerto.eu/public/statistiche/schemi4_h_en.html?. Gli autori presi in considerazione sono per lo più presi a caso.

${ }^{44}$ Vedi Glavičić, 2001: 21; 37.
} 
tiamo qui anche la posizione nell'emistichio posteriore dove la parola pesante appare solo una volta, traendosi dalla cesura centrale alla quinta dieresi (5D), 66: Affuit ece dies anniuersaria, Marti., mentre gli esempi della terza dieresi (3D) al quinto trocheo (5TR) non si trovano. Di solito nell'emistichio posteriore da Omero va collocata dopo la cesura centrale, scavalcando le $\mathrm{H}$ e B ed evitando la 3D, mentre nell'esametro latino inizia con la 3D e di solito va al 5TR, meno frequentemente alla $5 \mathrm{D}$, ed eccezionalmente alla quinta arsi (5J). ${ }^{45}$

\begin{tabular}{|c|c|c|c|c|c|c|c|c|c|}
\hline \multicolumn{10}{|c|}{ Tabella 3. Rapporto tra dattili e spondei negli 8 tipi più frequenti di esametro } \\
\hline \multicolumn{10}{|c|}{ Rapporto tra dattili e spondei negli 8 tipi più frequenti di esametro } \\
\hline autore & 1. & 2. & 3. & 4. & 5. & 6. & 7. & 8. & $\mathrm{D} / \mathrm{S}$ \\
\hline Vergilius & DSSS & DDSS & DSDS & SDSS & SSSS & DDDS & SSDS & SDDS & $12 / 20$ \\
\hline Ovidius & DDSS & DSSS & DSSD & DSDS & DDSD & DDDS & DSDD & DDDD & $20 / 12$ \\
\hline Claudianus & DSDS & DDSS & DSSS & SDSS & SSDS & DDDS & SDDS & DSSD & $13 / 19$ \\
\hline Cicero & DSSS & DDSS & SDSS & SSSS & DSDS & SDDS & DDDS & SSDS & $12 / 20$ \\
\hline Catull. & DSSS & SDSS & SSSS & DDSS & DSDS & DSSD & SSDS & DDSD & $12 / 20$ \\
\hline Lucr. & DSSS & DDSS & DSDS & SDSS & DDDS & DSSD & SSSS & DDSD & $14 / 18$ \\
\hline Germanicus & DSSS & DDSS & SDSS & DSDS & SSSS & DSSD & DDSD & SSDS & $12 / 20$ \\
\hline Manilius & DSSS & DDSS & SDSS & DSDS & SSSS & SDDS & DDDS & SSDS & $12 / 20$ \\
\hline Persius & DSSS & DDSS & DSDS & SDSS & DSSD & DDDS & DDSD & SSSS & $14 / 18$ \\
\hline Statius & DSDS & DSSS & DDSS & DDDS & DSSD & DSDD & DDSD & SDSS & $17 / 15$ \\
\hline Silius Ital. & DSSS & SDSS & SSSS & DSDS & DDSS & SSDS & SDDS & DSSD & $11 / 21$ \\
\hline Mart. & DSSS & DSDS & DDSS & DSSD & SDSS & DDSD & SDSD & DDDS & $16 / 16$ \\
\hline Auson. & DSDS & DSSD & DSSS & DDSS & DDSD & SSDS & DSDD & DDDS & $17 / 15$ \\
\hline Paul. Nol. & DSDS & DDSS & DSSS & DSSD & DDSD & DDDS & DSDD & SDSS & $17 / 15$ \\
\hline Cypr. Gall. & DSSS & DDSS & SDSS & DSDS & SSDS & SDDS & DDDS & SSSS & $12 / 20$ \\
\hline Ven. Fort. & DDSD & DDSS & DDDS & DDDD & DSDS & DSSD & DSSS & SDSD & $19 / 13$ \\
\hline Anthol. Lat. & DSSS & DDSS & DSDS & SDSS & DDDS & DDSD & DSSD & SSDS & $15 / 17$ \\
\hline Verronus & DSSS & DDSS & DSDS & DSSD & SDSS & DDDS & DDSD & SSDS & $15 / 17$ \\
\hline
\end{tabular}

Per quanto riguarda la tessitura del quarto piede, si nota la drastica riduzione del quarto piede omodino, che si verifica in Verronus appena il 24,51\% (302 versi). Già J. Knight ha sottolineato i diversi effetti ottenuti dall'eterodine (resistenza, difficoltà, sforzo, stanchezza, pathos) e dall'omodine (rapidità, levigatezza, libertà da moderazione $\mathrm{e}$ tensione) e ha dimostrato che Virgilio, a differenza della maggior parte dei poeti dell'esametro latino, in uguale maniera pre- e post-virgiliani, abbia ridotto la percentuale di omodine del quarto piede da cinquanta

${ }^{45}$ Vedi Glavičić, 2001: 22; 39. 
o sessanta a meno di quaranta. ${ }^{46}$ Verronus lo ha ridotto molto di più, ma ciò può essere giustificato dal tema principale del poema: il martirio, che ha momenti di pathos molto più numerosi che quelli della libertà di ritegno.

\section{Emistichio posteriore}

Ci sono due versi spondaici (0,16 \%), 558: Excidet, inque dies maius capit incrementum, senza alcuna pausa nell'emistichio posteriore, e questo verso non è affatto virgiliano, anzi, secondo il numero di esempi, neanche si potrebbe dire classico, ${ }^{47}$ ed il 1007: Lictoraque affectant flatu quae torret Iapyx:, che è spondaico virgiliano (cfr. Aen. $10,425 ; 11,899 ; 12,384)$, anche se è stato preceduto in questo da Lucrezio (cfr. rer. nat. 4, 932), e una tale struttura dello spondaico si può trovare anche in altri autori, come Stazio, Silo Italico (che tale struttura ha, come Virgilio, tre volte), oppure Paulino di Nola. ${ }^{48}$

Tuttavia Virg. ha una percentuale di spondaico più elevata: $0,24 \%$ (come Ovid.), e Verronus è situato affianco a Manil. e Petron. (entrambi con $0,16 \%$ ), ed è più vicino a Pers., Tert., Nemes., Mar. Victor. $(0,15 \%)$, anche a Lucan. con $0,17 \%$, o Cic. Arat. phaen. con $0,18 \%$ (mentre Cic. ha un totale di $0,27 \%$ ). Sia la struttura che le percentuali dimostrano chiaramente che il suo modello per il verso spondaico non era Virgilio. ${ }^{49}$

La principale incisione ritmica nell'emistichio posteriore è la quinta dieresi (5D) (734 versi, 59,57\%), seguita dal quinto trocheo (5TR) (601 versi, 48,78 \%), mentre le incisioni dopo la quinta (4 versi, $0,32 \%)$ e la sesta arsi (6 versi, $0,48 \%$ ) sembrano delle eccezioni.

\section{Cesure e dieresi}

La più comune a Verronus è la cesura centrale, la $\mathrm{P}$, che ricorre nell' $84,41 \%$ dei versi, seguita dalla H $(72,15 \%)$ e dalla T $(64,69 \%)$, ed una percentuale abbastanza alta tiene anche la B (57,79\%), come

${ }^{46}$ Duckworth, 1964: 42.

${ }^{47}$ Forse più precisamente a dire il hapax di Catullo, cfr. CATVLl. carm. 64, 67: Ípsiǔs

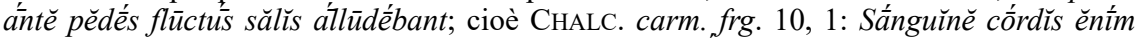

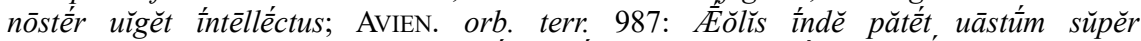

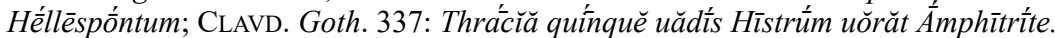

${ }^{48}$ Cfr. Stat. Theb. 2, 477; 10, 268, Sil. Ital. Pun. 4, 478; 6, 640; 10, 268, Nemes. cyn. 285, IVvenc. euang. 4, 63, Avson. epist. 22, 25, PAVl. Nol. carm. 19, 485, PAVL. PELL. euch. 3.

${ }^{49}$ Neanche imitò altri autori, pur ovviamente essendo di essi consapevole, perché erano, se non modelli di ruolo nella versificazione, almeno una notevole fonte di ispirazione in altri elementi versificativi. Le percentuali di versi spondaici in questi autori sono le seguenti: Sil. Ital. 0,08 \% (due volte tanto di Verronus), Stat. 0,05\%, Clavd. 0,06\%, PAVL. Nol. 0,49 \%, AVIEN. 1,75\%, ANTHOL. LAT. 0,1\%. Le tabelle per poter comparare i dati sono disponibili in rete: www.pedecerto.eu/public/statistiche/speciale4_H_en.html? e www.pedecerto.eu/public/statistiche/schemi4_H_en.html?. 
anche la $1 \mathrm{~J}(24,10 \%)$, il che semplicemente indica che Verronus, spesso, quasi in un quarto dei versi, inizia il suo esametro con una parola monosillabica. Il primo posto tra le cesure femminili occupa la 1TR $(15,34 \%)$, segue quella dopo il terzo trocheo (KT) $(14,28 \%)$, e poi dopo quello secondo (2TR) (11,76\%), mentre l'incisione dopo il quarto trocheo (4TR) si verifica solo in un piccolo numero di versi $(2,27 \%)$.

Nell'emistichio anteriore spicca al primo posto la 1D (41,39\%), seguita dalla 3D (13,47 \%), e dalla seconda dieresi (2D) (4,46\%).

La cesura centrale può essere anche sintatticamente contrasegnata, come ad esempio 5: Romanus Princeps: infracto ...; 14: Proiice vaniloquos: rebusque ...; 154: Coelicolum Regi, uultu ...; 178: Relligionis iter frustra ...; 218: Linquere coguntur celeres ...; 303: Excita praepetibus pennis .... Nella maggior parte dei casi $(80,92 \%)$ è accompagniata da una delle cesure laterali, ed il modello triplice $\mathrm{T}+\mathrm{P}+\mathrm{H}$ si trova in poco più di un terzo dei versi $(35,67 \%)$. In poco meno di una dozzina di versi il posto della H occupa la B $(\mathrm{T}+\mathrm{P}+\mathrm{B}: 8,76$ $\%$ ), o la 3D (T+P+3D: 8,11\%). Spesso questo modello viene esteso con la B $(\mathrm{T}+\mathrm{P}+\mathrm{H}+\mathrm{B}:$ 12,66 \%), o in aggiunta della $1 \mathrm{~J}, \quad \mathrm{D}$ o $1 \mathrm{TR}$ $(\mathrm{J} / 1 \mathrm{D} / 1 \mathrm{TR}+\mathrm{T}+\mathrm{P}+\mathrm{H}+\mathrm{B}:$ 7,54\%), come ad esempio, 55: Posse regi, casu nullo, nec tempore labi; 65: Per vicos Asiae cunctos, per castra, per urbes; 76: Floribus hi cumulant aras: hi thura ministrant; 104: Nunc ruit in praedam paribus de uertice pennis. Nel caso in cui la P non sia contrassegnata sintatticamente, lo può essere una delle cesure laterali, ad es. 163: Quaeue boni tanti uestra est iactura? quis auctor?; 179: Ludere nos blandis uerbis, tua munera scito; 325: Vrsa unum laniat Iииеnum, lea dentibus unum; come possono esserne più sintatticamente contrassegnate, ad es. 79: Conclamant homines, plausu, sonituque tubarum; 96: Idem animus, Regi solum, qui condidit orbem; 136: Extulerat: Nautae similis, qui sole cadente.

Il modello $\mathrm{T}+\mathrm{H}$ in tale forma si verifica solo tre volte (422: Exangues, uultu incedit facieque serena; 592: Incolumis, ferrique acies obtusa repente; 1176: Labentes fulcite animas, populosque docete), e si trova anche in modelli amplificati $\mathrm{D}+\mathrm{T}+\mathrm{H}$ (119: Munera nec sperno: imprimis redeuntibus annis $\left.{ }^{50}\right), \mathrm{J}+\mathrm{T}+\mathrm{H}$ (220: Grex ouium, nota inuitus regione recedit: $\left.{ }^{51}\right), \mathrm{J}+\mathrm{D}+\mathrm{T}+\mathrm{H}$ (1192: Hunc mala (sed more hostili) durissima uallo $\left.^{52}\right), 1 \mathrm{TR}+\mathrm{T}+\mathrm{H}$ (281: Docta queat superare, humiles

${ }^{50}$ Cfr. Verg. Aen. 9, 147: Áppărăt èt mēcum ínuādìt trěpidántǐă cấstra?; PAVL.

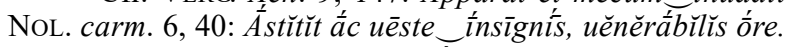

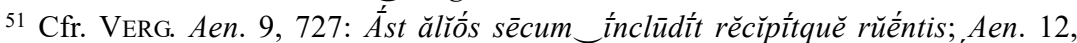

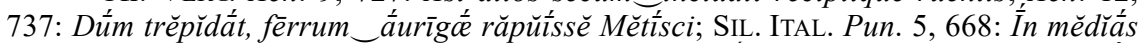

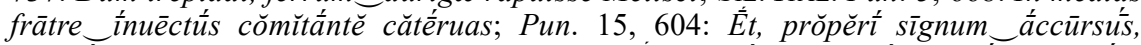

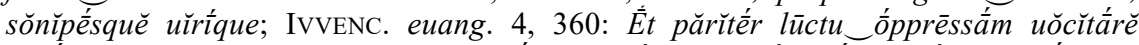

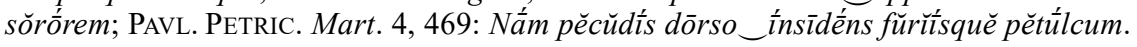

52 Verg. Aen. 8, 568: Nốn ĕgŏ nứnc dūlci ámplēxú dīuéllěrěr ứsquam; PAVL. Nol.

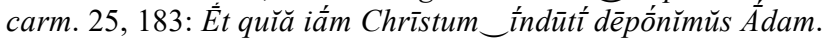


ingressus in aedes ${ }^{53}$; 405: Dente rotas, roseisque humeris conspersa cruore; 410: Quique tuis ultro astrigera succurris ab arce, $\left.{ }^{54}\right), \mathrm{J}+\mathrm{T}+\mathrm{H}+\mathrm{B}$ (26: Num cedit? grandem Almachi num diua furorem $\left.{ }^{55}\right), 1 \mathrm{TR}+\mathrm{T}+\mathrm{H}+\mathrm{B}$ (906: Munus obit, rerumque habiles sibi ducit habenas. ${ }^{56}$ ) e $\mathrm{J}+\mathrm{D}+\mathrm{T}+\mathrm{H}+\mathrm{B}$ (1189: Pars ea, quam cecini, aeterni sine numine Regis ${ }^{57}$ ).

La cesura dopo il terzo trocheo si verifica in 177 versi $(14,36$ $\%$, dei quali 128 volte parte dal più antico triplice modello $\mathrm{T}+\mathrm{KT}+\mathrm{H}$. Da questo numero poi nel modello semplice $\mathrm{T}+\mathrm{KT}+\mathrm{H}$ si verifica 21 volte, ad es. 40: Ingenium, fandique modus formidine torpet; (anche v. 97, 188, 190, 277, 291, 486, 491, 515, 557, 817, 907, 965, 1015, 1049, $1117,1120,1150,1158)$. In nessuno di questi versi non viene contrassegnata sintatticamente, ma lo sono o la $\mathrm{T}$ o la $\mathrm{H}$. Il modello può essere esteso con incisioni dopo la prima arsi o dopo il primo trocheo, $\mathrm{J} / 1 \mathrm{TR}+\mathrm{T}+\mathrm{KT}+\mathrm{H}$, ad es. 30: Quae terris superauit ouans Euphemia Virgo; 109: Solis equus, totamque tegit caligine nigra. Può anche essere esteso con la $\mathrm{B}$, e in aggiunta con la $1 \mathrm{~J}$ o $1 \mathrm{TR}$, ad es. 2: Mirificis descripta modis, ut fortia Poeni; 53: Quod Prisci posuere Deo, qui praesidet armis; 132: Trade neci; meritasque mihi det sanguine poenas.

La troviamo ulteriormente nel modello $\mathrm{T}+2 \mathrm{D}+\mathrm{KT}+\mathrm{H}$, anche esso estendibile con la 1J o $1 \mathrm{TR}$ e la B, ad es. 21: Alauro qui nomen habet Vincentius acres; 128: Prisce, tibi si cara mei reuerentia sacri; 210: Perpessi uos parta quies (uos) testor, Olympi; 585: Non gladius, non dira fames, non tela, nec ignes; 641: Sola negat: quam mente ferox quam pectore fortis. In un caso la $\mathrm{T}$ viene sostituita dalla 1D, 231: Pectus Virgineum: ille dolo ferroque paratus; e troviamo svilluppati anche tali modelli, o intagliati dalle incisioni ritmiche: $1 \mathrm{TR}+1 \mathrm{D}+\mathrm{P}+\mathrm{KT}+\mathrm{B}$ (761: Pectus in inuisum per acutum figite telum ${ }^{58}$ ) e $1 \mathrm{TR}+\mathrm{T}+\mathrm{P}+\mathrm{KT}+$ 3D+B (844: Inque decus probrum, uel in ocia tuta laborem: ${ }^{59}$ ).

La KT viene sintatticamente contrassegnata appena sette volte, che corrisponde a $3,95 \%$ di tutti i versi nei quali si verifica.

${ }^{53}$ Cfr. OvID. met. 8, 584: Tántŭs ěrám părĭtếrque_ănümís ìnmánĭs ĕt úndis; PAVL.

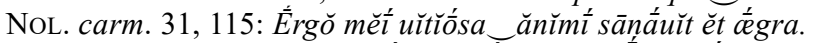

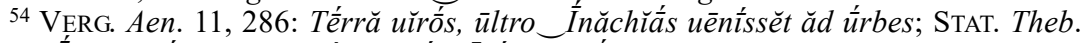

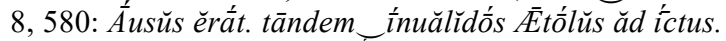

${ }^{55}$ NeMES. cyn. 264: Ěst ôllî́s quōdque ¿ínfrēnếs, quōd líběr ŭtếrque; ClaVD. in

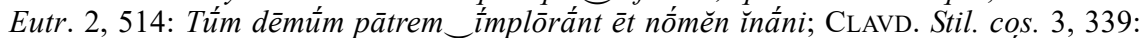

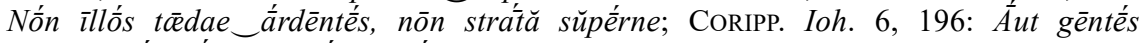
tāntum, ¿égēeúnt quāe bếllă prî̀res.

56 VERG. Aen. 2, 132: Iámquě diếs infánda ădĕrất; mĭhĭ sắcră părări; GERM.

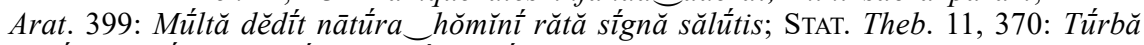
rŏgấnt āmbóéque_ăcǐés; rŏgăt íllă sướrum.

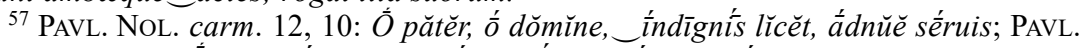

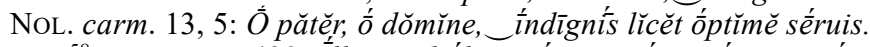

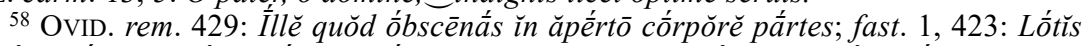

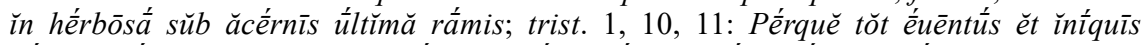
cốncŭtă uếntis; LAVs Pis. 63: Vếrbă nĕc ínclūsố sĕd ăpếrtō píngĕrĕ flóre; STAT. silu. 2, 3, 56: Spérăt ĕt ắmplēxús, sěd ăquárūm spírìtŭs ărcet.

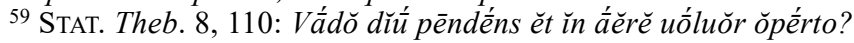




\begin{tabular}{|c|c|c|c|}
\hline & 8 & 0 & $\stackrel{\infty}{0}_{0}^{\infty}$ \\
\hline & 8 & $\nabla$ & ב్ \\
\hline & है & m & $\begin{array}{l}n \\
\text { ñ } \\
n^{2}\end{array}$ \\
\hline & $\stackrel{0}{p}$ & $\overrightarrow{8}$ & $\underset{\substack{\infty \\
\infty}}{\infty}$ \\
\hline & $\underline{E}$ & ㄱ & $\overrightarrow{\vec{A}}$ \\
\hline 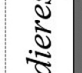 & 글 & $\stackrel{\curvearrowleft}{\Xi}$ & $\stackrel{\circ}{=}$ \\
\hline $\begin{array}{l}0 \\
0 \\
=\end{array}$ & $\cong$ & $\stackrel{\infty}{=}$ & 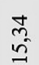 \\
\hline $\begin{array}{l}0 \\
0 \\
\end{array}$ & है & $\stackrel{\circ}{\circ}$ & $\stackrel{f}{\mathrm{f}}$ \\
\hline 8 & สิ & $n$ & $\underset{\substack{0 \\
\hdashline \\
\hdashline}}{ }$ \\
\hline s: & $\triangleq$ & $\stackrel{\circ}{n}$ & $\underset{\vec{F}}{\vec{F}}$ \\
\hline$\underset{D}{ \pm}$ & $\nabla$ & ลิ & $\stackrel{\circ}{\stackrel{+}{4}}$ \\
\hline 8 & $\oplus$ & $\stackrel{m}{=}$ & $\begin{array}{l}\text { s. } \\
\text { in }\end{array}$ \\
\hline & $\underline{z}$ & $\approx$ & 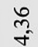 \\
\hline & $\equiv$ & $\underset{\infty}{\infty}$ & $\begin{array}{l}n \\
\text { dis }\end{array}$ \\
\hline & $A$ & 웅 & $\begin{array}{l}\vec{f} \\
\dot{\Phi}\end{array}$ \\
\hline & $F$ & ล̊ & $\begin{array}{l}\text { oे } \\
\text { : }\end{array}$ \\
\hline & & $\dot{z}$ & $\partial^{\circ}$ \\
\hline
\end{tabular}

La seconda dieresi si può trovare in modelli come $\mathrm{J}+\mathrm{T}+2 \mathrm{D}+\mathrm{P}+\mathrm{H}$ (99: Hic degunt ita $\mid$ que, et tacito sermone fruuntur $^{60}$; dove la sinalefe provoca la 2D), $\mathrm{T}+2 \mathrm{D}+\mathrm{P}+\mathrm{H}$ (ad es. 411: Supplicibus me nunc manibus pedibusque reuinctam ${ }^{61}$; come anche in $452,564,930$, e il modello es-

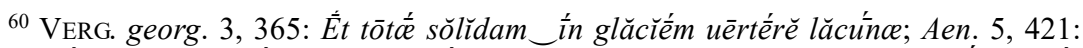

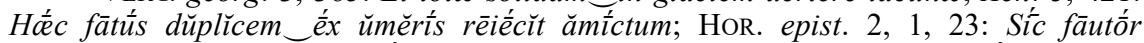

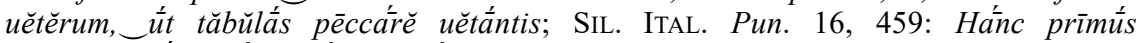
gălěam—(hấc ăciếs tērrếbăt Hǐbèras.

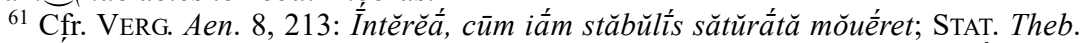

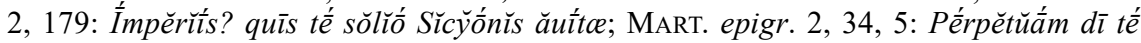


teso con la B 729: Iniiciat: quis non specie (uel saxea gestet;), come anche il modello $\mathrm{T}+2 \mathrm{D}+\mathrm{B}$, con la $2 \mathrm{D}$ sintatticamente contrassegnata (196: Contundi iubet; et uinciri compede crura), oppure $1 \mathrm{TR}+\mathrm{T}+2 \mathrm{D}+$ P+4TR (177: Sacra: dolus capit haec, uerumque (fatemur) aberrant ${ }^{62}$ ). Troviamo anche due esempi del modello $\mathrm{T}+2 \mathrm{D}+\mathrm{H}$ (estendibile con la B), 1230: Vulneribus; quae cladem animis mortemque minantur; 430: Terribiles non ira oculos, non uerba minantis ${ }^{63}$. La seconda dieresi viene sintatticamente segnata solo nell'esempio citato, altrove lo sono la cesura centrale oppure una delle cesure laterali.

Di conseguenza, Paschalis Verronus ha una media di almeno 4 incisioni ritmiche nell'emistichio anteriore $(4,06)$, la dieresi bucolica è un'incisione ritmica quasi regolare, che spesso accompagna la cesura semisettenaria, e l'emistichio anteriore viene in termini di ritmo spesso spezzato con cinque, sei o sette incisioni in modelli come $\mathrm{D}+\mathrm{T}+\mathrm{P}+$ $\mathrm{KT}+\mathrm{B}, \mathrm{J}+\mathrm{D}+\mathrm{P}+3 \mathrm{D}+\mathrm{H}+\mathrm{B}, \mathrm{J}+\mathrm{T}+\mathrm{P}+3 \mathrm{D}+\mathrm{H}+\mathrm{B}, \mathrm{J}+\mathrm{D}+2 \mathrm{TR}+\mathrm{P}+\mathrm{H}+\mathrm{B}, \mathrm{o}+\mathrm{J}+2 \mathrm{TR}+$ $\mathrm{P}+3 \mathrm{D}+\mathrm{H}+\mathrm{B}$.

\section{La tipologia del verso}

Risultati più che interessanti possono essere ottenuti con la ricerca della tiplogia del verso. Tale confronto, reso possibile dalla tecnologia moderna, offre, almeno in questo caso, opportunità di intuizione che non abbiamo avuto prima, ulteriormente stimolanti per nuove ricerche. Vale a dire, ora è possibile ottenere dati sulla frequenza del tipo di un singolo verso in un corpus che attualmente conta oltre 240 mila versi (213444 nell'analisi di tipologia di verso), tenendo conto non solo della metrica esterna, ma anche di quella interna.

Quindi poco più di un quarto di versi di Verronus $(25,8 \%)$ sono versi che compaiono nella forma esatta fino a 25 volte, ma in realtà sono tutti versi diversi, se si guarda la struttura della metrica interna. Ad esempio, 555: Saxa tuis, quae iure Deus sibi postulat a te, è un DSDD, nonché come 844: Inque decus probrum, uel in ocia tuta laborem, ma nel primo caso abbiamo $1 \mathrm{TR}+\mathrm{T}+\mathrm{P}+3 \mathrm{D}+\mathrm{H} / 5 \mathrm{TR}$, e in quest'ultimo $1 \mathrm{TR}+\mathrm{T}+\mathrm{P}+\mathrm{KT}+3 \mathrm{D}+\mathrm{B} / 5 \mathrm{TR}$. Poco più della metà dei versi $(50,8 \%)$ sono quelli che compaiono nel corpus fino a 100 volte.

\section{Versus Verroniani}

$\mathrm{Ci}$ imbattiamo in 25 versi che potremmo, in base alla situazione attuale, chiamare "Verroniani". Vale a dire, questi sono quelli che non si trovano nel corpus di nessun altro autore.

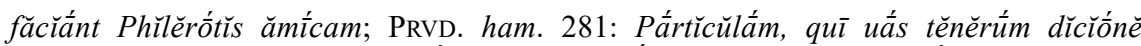

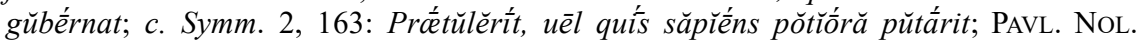
carm. 20, 404.

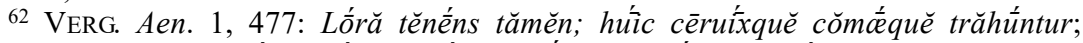

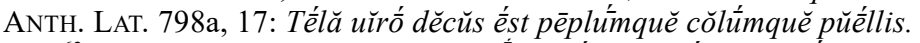

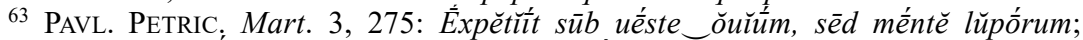

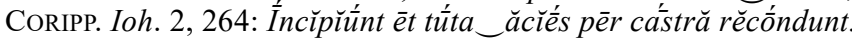


66: Affuit ecce dies anniuersaria, Marti (DDSS: D+2TR+P / 5D); 90: Foeda ministeria, effigies et sacra Deorum (DDDS: 1TR+H+B / 5TR); 164: Eia age, neglectos una instauretis honores, (DSSS: D+P / 5TR); 184: Fulgore, inque domos paucas dimisit in horas: (SDSS: $2 \mathrm{TR}+\mathrm{P}+\mathrm{H} /$ 5TR+5D); 199: Supplicia, atque amens animum circum omnia uertit: (DSDS: $\mathrm{P}+\mathrm{H}$ / 5D); 231: Pectus Virgineum: ille dolo ferroque paratus (SDDS: $\mathrm{D}+\mathrm{KT}+\mathrm{H} /$ 5TR); 389: Praesidis ad sedes, prauique ante ora Tyranni: (DSSS: $\mathrm{D}+\mathrm{T}+\mathrm{P} /$ 5TR); 440: Vrit, et extingui nequit, ustos nec sinit unquam (DSDS: $1 \mathrm{TR}+\mathrm{D}+\mathrm{P}+3 \mathrm{D}+\mathrm{B} / 5 \mathrm{~J}+5 \mathrm{D}$ ); 517: Ecce tibi in stadium ueniunt fera corpora, nudos (DDDD: $1 \mathrm{TR}+\mathrm{T}+\mathrm{P}+\mathrm{H}+\mathrm{B} / 5 \mathrm{D}$ ); 637: Diique Deaeque omnes per quos commissa luuntur (DSSS: $1 \mathrm{TR}+\mathrm{T}+\mathrm{P}+3 \mathrm{D}+\mathrm{H} /$ 5TR); 675: Munus agunt: iterum de Virgine Apellius audax (DDSD: 1TR+T+P+3D / 5D); 793: Hic modo supplicio immani torqueris, et igne, (DDSS: J+D+H / 5TR+5D); 836: Perpetuo hic patitur sacrum miserabilis ignem (DDSD: T+P+H / 5D); 841: Sed seruasse Dei praecepta, Euphemia, nec non (SDSS: $\mathrm{J}+\mathrm{D}+2 \mathrm{TR}+\mathrm{P} / 5 \mathrm{D}+6 \mathrm{~J}$ ); 877: Miles ab Eoo, et late sibi regna subegit: (DSSD: $1 \mathrm{TR}+\mathrm{D}+\mathrm{P}+\mathrm{H}+\mathrm{B} /$ 5TR); 878: Hinc datur Illiricis: Pataui de hinc fertur in Vrbem (DDDS: $\mathrm{J}+\mathrm{D}+\mathrm{P}+\mathrm{H}+\mathrm{B} / 5 \mathrm{TR}+5 \mathrm{D}$ ); 937: Fluminis exigui, hic summa ad fastigia surgit (ddss: $\mathrm{d}+\mathrm{p}+\mathrm{h} / 5 \mathrm{~d}$ ); 955: In monte is moritur, de monte ad sidera coeli (SDSS: $\mathrm{J}+\mathrm{T}+\mathrm{P}+\mathrm{B} /$ 5D); 1053: Nomen, at hic sacra qui fulget in aede Pyropus, (DSSD: 1TR $+\mathrm{D}+\mathrm{T}+\mathrm{P}+$ 3D+4TR+B / 5TR); 1057: Vera cutis, (mirum) neruos est cernere, et ungues (DSSS: $1 \mathrm{TR}+\mathrm{T}+\mathrm{P}+\mathrm{H}+\mathrm{B} / \mathrm{5D}$ ); 1099: Indicat, ipse rudi quam effingere carmine conor: (DDSD: $\mathrm{D}+2 \mathrm{TR}+\mathrm{P}+\mathrm{B} /$ 5D); 1128: Quam queat ingenium nostrum meminisse, sed heu me (DDSD: $\mathrm{J}+\mathrm{D}+\mathrm{P}+\mathrm{H} /$ 5TR+5D+6J); 1129: Cur iuga non licuit superare Heliconis, et undis (DDDD: J+D+T+P / 5TR+5D); 1149: Praeterea obscurum non est parientibus illam (DSSD: P+3D+H / 5D); 1160: Grando lapsa hominum fregit, pecudumque labores: (SDSD: $\mathrm{D}+\mathrm{P}+\mathrm{H} / 5 \mathrm{TR}$ ).

\section{Versus hapaces}

Versi che sono unici per ogni autore nel suo corpus, e non è molto probabile che Verronus abbia accidentalmente usato il tipo di verso esatto, si verificano così: i più rappresentati sono OVID. (met.) e STAT. (Theb.), ciascuno con tre esempi di verso unico, e PAVL. NOL. con due, mentre con un esempio si trovano: Sil. ITAL., AVSON., AVIEn., APVL., VEN. ForT., CYPR. GAll., HOR., PRVD. i EPIGR. Bob.

17: In Christi Athletas; quae contra his parta trophaea ${ }^{64}$ (SSSS: $\mathrm{J}+\mathrm{P}+3 \mathrm{D}+\mathrm{B} /$ 5TR); 27: Caecilia expauit? num formidauit acutos ${ }^{65}$ (DSSS: P+3D / 5TR); 91: Exosa a teneris, cupiensque a munere turpi ${ }^{66}$ (SDDS: $\mathrm{T}+\mathrm{P}+\mathrm{B} /$ 5D); 93: Abdidit, et celebri a populo se limine sepsit. ${ }^{67}$ (DDDS: $\mathrm{D}+\mathrm{T}+\mathrm{P}+\mathrm{H}+\mathrm{B} / 5 \mathrm{D}) ; 103$ : Accipiter: sese nunc ales ad aethera

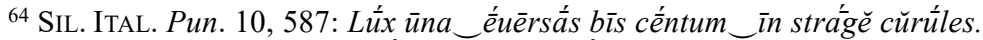

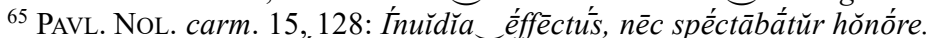

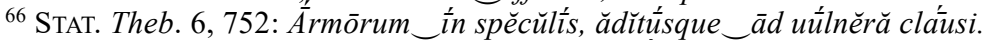

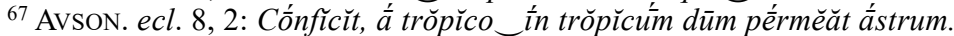


condit; ${ }^{68}$ (DSSD: $\mathrm{T}+\mathrm{P}+3 \mathrm{D}+4 \mathrm{TR}+\mathrm{B} /$ 5D); 317: Vincere, et illaesum tutari pectus ab hoste. ${ }^{6}$ (DSSS: $\left.\mathrm{D}+\mathrm{P}+\mathrm{B} / 5 \mathrm{TR}+5 \mathrm{D}\right) ; 333$ : Cernere erat puteo (quid non molitur iniqua ${ }^{70}$ (DDSS: $\mathrm{T}+\mathrm{P}+3 \mathrm{D}+\mathrm{H} / 5 \mathrm{TR}$ ); 555: Saxa tuis, quae iure Deus sibi postulat a te: $:^{71}$ (DSDD: $1 \mathrm{TR}+\mathrm{T}+2 \mathrm{D}+\mathrm{KT}+\mathrm{H}+\mathrm{B} /$ $5 \mathrm{D}+6 \mathrm{~J}$ ); 579: Immitti, ut puteum deiectum corpus in altum ${ }^{72}$ (SDSS: $\mathrm{T}+\mathrm{P}+\mathrm{B} / 5 \mathrm{TR}+5 \mathrm{D})$; 649: Erigite: hanc operam poscenti, hunc quaeso laborem $^{73}$ (DDSS: $\left.\mathrm{D}+\mathrm{T}+\mathrm{P}+\mathrm{B} / 5 \mathrm{TR}\right)$; 667: Incubuere locis iam Chalcedonis, et Vrbi: ${ }^{74}$ (DDSS: 2TR+P+3D / 5TR+5D); 830: Causa: beat splendor diuini luminis, in quem ${ }^{75}$ (DSSS: $\left.1 \mathrm{TR}+\mathrm{T}+\mathrm{P}+\mathrm{B} / 5 \mathrm{D}+6 \mathrm{~J}\right)$; 844: Inque decus probrum, uel in ocia tuta laborem: ${ }^{76}$ (DSDD: $1 \mathrm{TR}+\mathrm{T}+\mathrm{P}+\mathrm{KT}+3 \mathrm{D}+\mathrm{B} /$ 5TR); 856: Quod Virgo haec tribuit precibus quaesita uel ultro: ${ }^{77}$ (SDDS: $\mathrm{J}+\mathrm{T}+\mathrm{P}+\mathrm{H} /$ 5TR $+5 \mathrm{D}$ ); 887: Quo rapit immemorem uersus me? quo feror amens? ${ }^{78}$ (DDSS: $\mathrm{J}+\mathrm{D}+\mathrm{P}+\mathrm{H}+\mathrm{B} / 5 \mathrm{~J}+5 \mathrm{D}$ ); 936: Naiades hoc tantum Bradani priuantur honore ${ }^{79}$ (DSSD: $\mathrm{D}+\mathrm{T}+\mathrm{P}+\mathrm{H} /$ 5TR); 1096: Phidiacum ingenium, nec non mirabilis artem $^{80}$ (DDSS: $\left.\mathrm{P}+3 \mathrm{D}+\mathrm{H} / 5 \mathrm{D}\right)$.

\section{Versus authentici}

Versi che si verificano nel corpus da due a dieci volte. Nel numero dei modelli più rari si trovano VERG., SiL. ITAL., VAL. FLACC., Ovid., Manil., IVV., PAVl. Petric., Gratt. e LVCr., e in totale in questa categoria è il più rappresentato Virgilio, mentre il secondo posto, con un significativo numero di versi, tiene Lucrezio, seguito da Ovidio.

\section{Vergiliani versus}

Degli esempi più rari si individuano dalla Vita i seguenti: 119: Munera nec sperno: imprimis redeuntibus annis ${ }^{81}$ (2); 177: Sacra: dolus capit haec, uerumque (fatemur) aberrant ${ }^{82}(2)$; 410: Quique tuis ultro astrigera succurris ab arce, ${ }^{83}(2) ; 722$ : Sanguine purpureo, manet illa

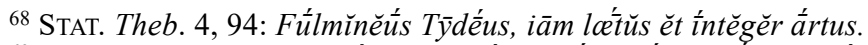

${ }^{69}$ AvIEN. orb. terr. 1381: Lìtŏre, ¿ét éxtrēmá térrárūm uìctŏr ìn óra.

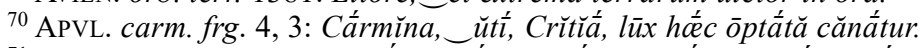

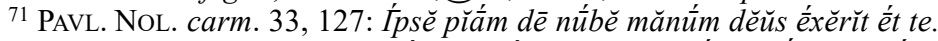

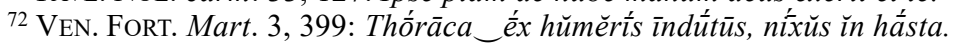

${ }^{73}$ OviD. met. 2, 223: Díndy̆măque èt My̆călè nātúsque àd sácră Cĭthớron.

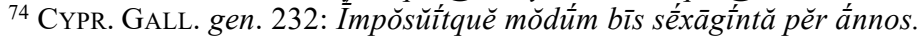

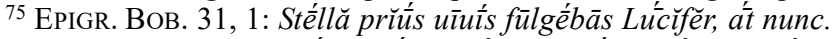

${ }^{76}$ STAT. Theb. 8, 110: Vádŏ dĭú pēndéns ět ìn áéré uó̀luŏr ŏpérto?

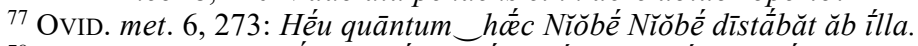

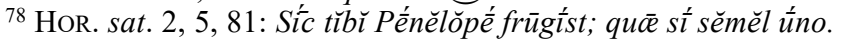

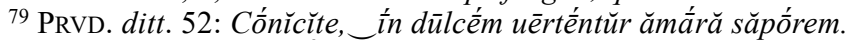

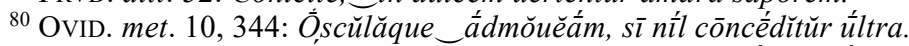

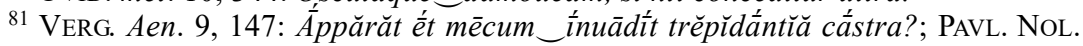
carm. 6, 40 .

82 Verg. Aen. 1, 477: Lốră těnếns tămĕn; huî̀c cēruî́xquĕ cŏmóequĕ trăhüntur; ANTH. LAT. 798a, 17.

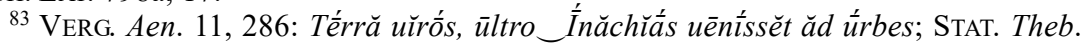
8,580 . 
immota flagellis, ${ }^{84}$ (2); 800: Vt morbo affecti, uitaque extrema ferentes: ${ }^{85}$ (2); 840: Inque Deum inque homines exactae crimina uitae: ${ }^{86}$ (2); 875: Corpus in his igitur patriis habitauit in ulnis ${ }^{87}$ (2); 1192: Hunc mala (sed more hostili) durissima uallo ${ }^{88}$ (2). Vanno qui aggiunti anche i versi 794, 906, 1133, 99, 160, 402, 194, 464, 867, 1010, 46, 714, 726, 220, 383, 411, 645, 1068, 1163, 1194, 758, 1102, 89, 234, $278,447,730,782,1187,1231,422,1170$.

\section{Ovidiani versus}

Degli esempi più rari si individuano dalla Vita i seguenti: 186: Parentes iussis, cum iam repetetis in imo ${ }^{89}$ (2); 281: Docta queat superare, humiles ingressus in aedes, ${ }^{90}(2) ; 1033$ : Littus, et in templis hic uota oblata rependunt..$^{91}$ (2). Vanno qui aggiunti anche i versi 512 , $918,1126,370,448,845,122,167,632,553,677,917,376,695,168$, 475.

\section{Lucretiani versus}

Degli esempi più rari si individuano dalla Vita i seguenti: 1188: Quod delata tui sit sancti Corporis ad nos ${ }^{92}$; e vanno aggiunti anche i versi ad es. 23, 41, 62, 95, 116, 320, 347, 723, 930, 580, 123, 158, $331,593,1077,262,435,652,989,623,698,828,1028,1043,1112$, come il noto modello di Lucrezio Aeneadum genetrix, ..., modello imitato da Verronus nel suo verso 516: Diriperent Iиuenum, speciem spectate triumphi.

In questi esempi rari trovano il loro posto ancora HoR., ad es. Euph. 525: Exhalantque animas: terris uolat astra secunda ${ }^{93}$; 564: Angelicis, tamen hanc fato cecidere Ministri ${ }^{94}$; 1091: Praeclaris Venetum, nunc tecto fixa sub alto ${ }^{95}$; 342: Brachia dant animos: hinc flamma

${ }^{84}$ Verg. Aen. 3, 488: Cốniŭgŭs Hếctŏrěcé. căpĕ dốna ēxtrếmă tŭórum; OvID. met. $11,173$.

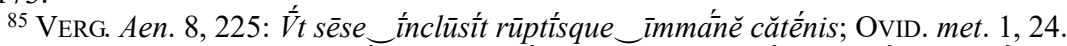

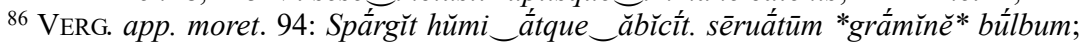
PAVL. PELl. euch. 297.

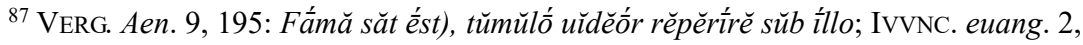
259.

${ }^{88}$ Verg. Aen. 8, 568: Nốn ĕgŏ núnc dūlci_ámplēxú dīuétlěrěr úsquam; PAVL. Nol. carm. 25,183 .

${ }^{89}$ OviD. met. 13, 90: Fớrtūnấm pūgnớ, nōn súm süpěrấtŭs ăb îllo; Pont. 2, 8, 57.

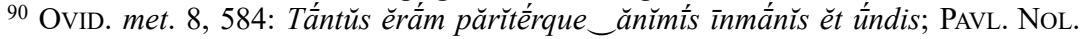
carm. 31,115 .

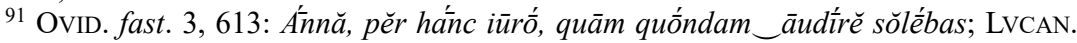
Phars. 9, 563.

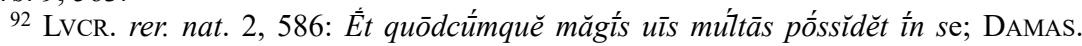
carm. 66, 4 .

${ }^{93}$ Hor. sat. 2, 5, 60; Prop. eleg. 2, 1, 71; Ovid. met. 15, 36; StAT. Theb. 9, 28; Ivv. sat. 10, 333; PAVll. Pell. euch. 251; PAVL. Petric. Mart. 3, 430.

${ }^{94}$ Hor. ars 223; Ovid. met. 6, 296; Sil. ItAl. Pun. 8, 652; 14, 665; PAVl. Nol. carm. 21, 415; frg. epist. 8, 101; ENNOD. carm. 2, 104, 9.

${ }^{95}$ Hor. sat. 2, 6, 30; LvCAN. Phars. 3, 655; 7, 600; STAT. silu. 3, 4, 81; MAR. VICTOR aleth. 2, 56; SIDON. carm. 7, 231; PRISC. periheg. 590. 
extrema minatur ${ }^{96}$; 1005: Ignarus pretium quantum ferat ipse per altum: ${ }^{97}$; poi CATVLL., ad es. Euph. 905: (Sic nostri docuere senes, quis credere fas est $)^{98}$; uguale nei v. 75, 94, 558, 1071; MANIL., ad es. Euph. 246: Commoneo, ac animo Marti fer liba uolenti, ${ }^{99}$; (uguale nei v. $\left.791^{100}, 591,130,815,365,902\right)$; STAT., ad es. Euph. 227: Egressus Priscus de bello, et caede recenti, ${ }^{101}$; (uguale nei v. 1069, 100, 1061, 325, 1060); SIL. ITAL., ad es. Euph. 11: Tuque addicta sacris, Regique dicata superno ${ }^{102}$; (uguale nei v. $282^{103}, 864^{104}, 1088^{105}$, 1046); PROP., ad es. Euph. 622: Ire ad Ditis opem, consuetaque quaerere fallax ${ }^{106}$; (con i v. $745^{107}, 214^{108}$ ); VAL. FLACC., ad es. Euph. 126: Ritu exculta cadent, pietasque ueterrima gentis ${ }^{109}$, (e il v. $1090^{110}$ ).

Con un esempio di tipo specifico, completano l'immagine IVV., Euph. 285: Me misit solio, ut magnam uim uiribus addam, ${ }^{11}$; PAVL. PETRIC., Euph. 430: Terribiles non ira oculos, non uerba minantis ${ }^{112}$; GRATT., Euph. 1113: Ingressae effigiem uenerantur, et oscula figunt. ${ }^{113}$; PAVL. Nol., Euph. 1189: Pars ea, quam cecini, aeterni sine

${ }^{96}$ Hor. sat. 1, 8, 40; VAL. Fl. Argon. 7, 360; Sil. Ital. Pun. 2, 436; 8, 127; 16, 523; IVv. sat. 9, 128; MAR. VICTOR aleth. 1, 71; 1, 469; aleth. 1, 480; ANTH. LAT. 931, 43.

${ }^{97}$ Hor. epist. 1, 10, 4; MANIL. astr. 1, 571; 2, 236; Stat. Theb. 11, 741; Sil. ItAL. Pun. 16, 9; AVIEN. Arat. 866; PAVl. Nol. carm. 18, 104; SedVl. carm. pasch. 1, 208; ANTH. LAT. 21, 111; 726, 26.

${ }^{98}$ CATVll. carm. 68, 41; PRVD. c. Symm. 2, 307; PAVL. Nol. carm. 21, 786.

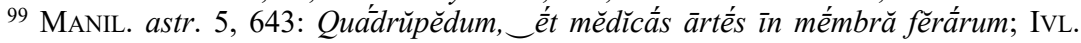
TOLET. epitaph. 1, 3 . 56.

100 MANIL. astr. 2, 553: Céntāurum ềt Pīscếs ēt tề, Căpricoórně, rügếntem; astr. 3,

${ }^{101}$ Stat. Theb. 3, 94; PAVl. Petric. Mart. 2, 703; Alc. Avit. carm. 2, 148.

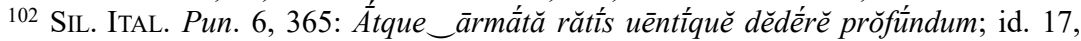
150.

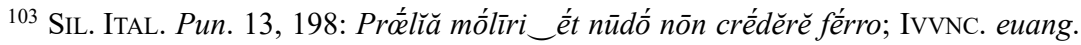
2,810 .

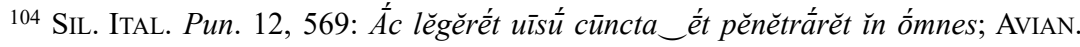
fab. 29,9 .

${ }^{105}$ Sil. ITAL. Pun. 10, 37: Nấm Lăbǐênŭs ŏbìt pěnĕtrắntě pĕr íllĭă cốrno; PAVL. Nol. carm. 20, 102.

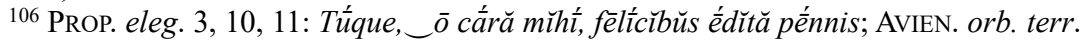
1355; PRVD. c. Symm. 2, 867.

${ }^{107}$ Prop. eleg. 3, 13, 17; Ovid. epist. 21, 213; LvCAn. Phars. 2, 16; VAl. Fl. Argon. 3, 8; Stat. silu. 1, 5, 29; Sil. ItAl. Pun. 17, 121; Avien. orb. terr. 1316; PaVl. Nol. carm. $26,169$.

${ }^{108}$ Prop. eleg. 1, 9, 25; 2, 1, 3; OvID. am. 3, 6, 45; OvID. rem. 221; epist. 20, 237; Pont. 1, 3, 9; Hil. Pict. Macc. 168; Clavd. carm. min. 17, 41; ANTH. Lat. 17=Hos. Getae Med., 3.

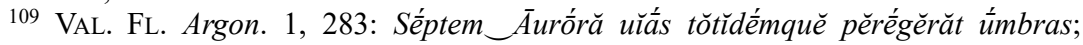
MART. epigr. 7, 58, 3 .

110 VAL. Fl. Argon. 3, 165; Sil. Ital. Pun. 15, 106; Sidon. carm. 5, 371.

${ }^{111}$ Ivv. sat. 11, 96: Séd nūdồ lătěre _èt pāruî́s frōns ấrěă léctis; PROBA cento 524.

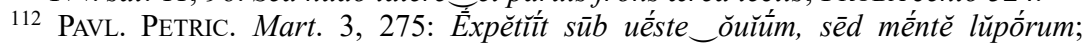
CORIPP. Ioh. 2, 264.

113 GRATT. cyneg. 355: Vî́rōsam_élŭuīếm lăcěrî́quĕ pěr úlcěrĭs ốra; PRVD. ham. 821. 
numine Regis ${ }^{114}$; LVCIL. (Euph. 44: Bizantinorum, muris turrita superbis, ${ }^{115}$ ), e come versi Luciliani si trovano anche ad es. Euph. 189, 225, 494, 522, 532, 692, 751, 769, 838, 903 e 934; LVCAN. (Euph. 65 ${ }^{116}$, $257^{117}$ ); SEN. (Euph. 289 ${ }^{118}$ ), NEMES. (Euph. $26^{119}$ ) e ILIAS LAT. (Euph. $\left.922^{120}\right)$.

Degli altri autori, per vedere la larghezza e ampiezza di testi e autori studiati da Verronus, citeremo ACC. (Euph. 490: Incumbunt operi flamma crepitante recentes ${ }^{121}$, 526, 702, 1039, 1200, 1206), VARR. (Euph. 527: Cum rursus mittat magno super astra Colono ${ }^{122}$; 540, 668, 899, 997, 1029), TIBVLL. (Euph. 585: Non gladius, non dira fames, non tela, nec ignes $^{123}$ ), LVTAT. (Euph. 822: Sacra gerit manibus speratae praemia pugnae ${ }^{124} ; 1135^{125}$ ), BIBAC. (Euph. 1042: Sacra colunt, quatiuntque poli conuexa frequentes ${ }^{126}$ ), ma anche SVEIVS (Euph. 984: Numinibusque suum numen consortibus addit: $\left.{ }^{127}\right)$, e LIV. ANDR. (Euph. 589: Haec ubi dicta dedit direxit lumina coelo; 963) ${ }^{128}$.

Non è affatto trascurabile il numero di versi originariamente ciceroniani (ad es. Euph. 314: Incolumes artus cernit, ruptosque rigen$t i)$, sebbene in seguito furono abbondantemente usati come modelli di

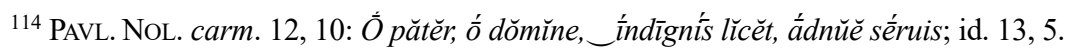

115 LVCIL. sat. 163; 180; VerG. app. dirae 139; DrAC. laud. dei 3, 659; ANTH. LAT. $611,3$.

116 Lvcan. Phars. 9, 122; Stat. Theb. 12, 188; Sil. ItAl. Pun. 9, 584; 14, 469; AviEn. orb. terr. 1224; ClaVD. in Eutr. 2, 402; Stil. cos. 1, 291.

117 Lvcan. Phars. 10, 497; Stat. Ach. 1, 299; Sil. Ital. Pun. 2, 153; Clavd. Hon. IV cos. 228; PrVD. psych. 76; ProsP. ingrat. 454; SidOn. carm. 7, 481; ANTH. LAT. 8, 103.

118 Sen. epigr. 2, 5; Stat. Theb. 10, 612; 11, 484; Sil. ItAl. Pun. 11, 162; 11, 340; MAR. ViCT. aleth. 3, 138; 3, 716; PAVL. PELl. euch. 124.

${ }_{119}$ NEMES. cyn. 264: Ëst ōllìs quōdque ¿ínfrēnếs, quōd líbèr ütếrque; Clavd. in Eutr. 2, 514; Stil. cos. 3, 339; CORIPP. Ioh. 6, 196.

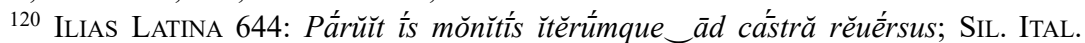
Pun. 2, 44; 15, 197; AMBR. tern. num. 4; VEN. FORT. carm. 5, 5, 45.

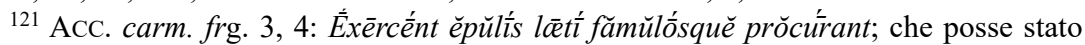
tratto anche da LVCR. (rer. nat. 2, 611; 5, 1212; 5, 1230), oppure VERG. (ecl. 1, 2; Aen. 3, $129 ; 6,248 ; 7,57 ; 8,208 ; 10,293 ; 10,376 ; 12,934)$.

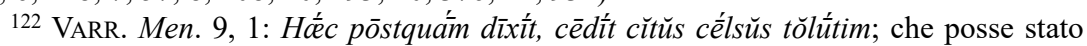
tratto anche da LVCR. (rer. nat. 4, 417; 4, 1075), o VERG. (georg. 1, 113; Aen. 3, 145), o altri autori (Ovid., STAT., Sil. ITAL., ...?).

123 TiB. eleg. 1, 3, 47: Nớn ăcüés, nōn t̀ră fǘt, nōn bếllă, nĕc énsem; anche possibilmente tratto da autori successivi.

${ }^{124}$ LvTAT. epigr. 2, 3: Pácě mŭhí lìcéăt, cāelếstēs, dícérě uếstra; anche possibilmente tratto da autori successivi.

${ }^{125}$ LVTAT. epigr. 2, 3; anche possibilmente tratto da autori successivi.

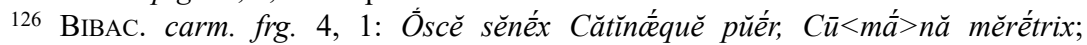
probabilmente tratto da autori successivi.

127 SveIvs carm. frg. 1, 7: Díssěrǔérě, nŏuốs frūctús mōrtálǐbŭs dántes; anche probabilmente tratto da autori successivi.

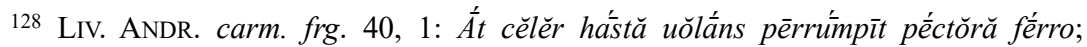
probabilmente tratto da LVCR., VERG., OVID., o qualcuno degli autori successivi. 
verso da autori successivi ${ }^{129}$, con un numero di versi assai considerevole (153 o il 12,41\%) che sono stati originariamente usati da Ennio, come ad es. Euph. 185: Haec addens ne uana fides, uos credo futuros $^{130}$; o 211: Astra uocant: pugnate Viri certamen ouantes ${ }^{131}$; e così via, versi per i quali avremmo prima di tale ricerca supposto che fossero da Verronus stati presi da LVCR., VERG., OVID. o qualuno dei classici successivi, ma a questo punto oseremo dire che è molto probabile che Verronus era consapevole che tali versi erano originariamente enniani.

\section{Conclusione}

Che Paschalis Verronus conoscesse bene non solo gli autori classici, ma anche quelli postclassici, così come quelli cristiani, si può chiaramente vedere da tutte le peculiarità trovate nel suo poemetto e discusse ed analizzate in questo articolo. Che Virgilio abbia lasciato su di lui un influsso chiaro e visibile, sia letterario che versificativo, è logico e abbastanza compensibile. Ma, soprattutto in termini di modelli versificativi, solo lo studio della metrica interna fornisce un quadro migliore di Virgilio come un modello di ruolo seguito da Verronus nella costruzione del verso, considerando che le differenze che si verificano in termini di metrica esterna non offrono un argomento valido, cioè indicano un influsso virgiliano minore. Si potrebbe dire che la Vita è stata composta sul principio: "un po' di tutto, avendo sempre Virgilio vicino". Inoltre, Verronus ha costruito il suo poemetto combinando alternativamente schemi noti e frequenti, con quelli meno noti, cioè con i suoi, originari d'autore. Questo fatto ha gettato una nuova luce sull'autore spiegando il perché questa breve epopea agiografica è in certe parti ruvida all'orecchio, o, come abbiamo già evidenziato precedentemente, gravata da ripetizioni inutili, il che la rende noiosa e faticosa. Per quanto la Vita non sia, in certe parti, uno scritto letterario-artistico geniale, Di Pasquale gli ha negato ogni particolare valore letterario, dando maggiore rilevanza al messaggio religioso, in base ai dati agiografici. A nostro avviso non è questo ciò che rende speciali $\mathrm{i}$ versi di Verronus, ma questa particolarità si manifesta con troppo sforzo impiegato nella versificazione stessa. Essa è la dimostrazione di tutta l'ampiezza dello spettro dell'esperienza acquisita dal nostro autore, e conferma, in larga misura, la tesi accennata da Di Pasquale, di uno studio appassionato dei classici. Probabilmente Verronus avrebbe potuto compilare meglio la Vita, opera di medio valore letterario, nella quale ha dimostrato la capacità di usare non solo le principali

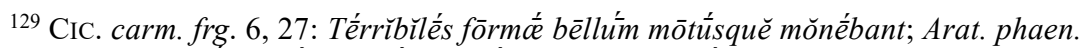
fragm. max. 178: Spinngèrám sūbtếr cāudám Pīstrícŭs ădhớsit; e dopo questo tipo viene abbondantemente usato da LVCR., VERG., OVID., MANIL., LVCAN. itd.

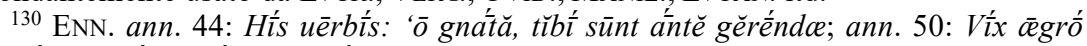
cūm cốrdě méó mē sốmnŭs rělíqquit.

${ }^{131}$ ENN. ann. 280: Suắŭ̌s hŏmố, iūcúndŭs, sǔố cōntếntŭs, běátus. 
soluzioni versificative, ma anche quelle meno conosciute. In questo senso, Verronus ha composto il suo poemetto più professionalmente che artisticamente, oggi potremmo dire che sia stato composto in modo scientifico, e il valore dell'opera sta proprio nell'equilibrio dei suoi modelli di ruolo versificativi, da quelli generalmente noti, a quelli rari, fino a quelli d'autore originari.

\section{BIBLIOGRAFIA:}

Di Pasquale, Nicolino. Vita di Sant'Eufemia Vergine e Martire di Calcedonia. Irsina, Editrice BMG Matera, 2001.

Duckworth, George E. "Variety and Repetition in Vergil's Hexameters". Transactions and Proceedings of the American Philological Association, (1964), 9-65.

Duckworth, George E. Vergil and Classical Hexameter Poetry: A Study in Metrical Variety. Ann Arbor, University of Michigan Press, 1969.

Glavičić, Branimir. Versifikacija hrvatskih latinista. Split, Književni krug, 2001.

Ianora, Michele. Memorie storiche, critiche e diplomatiche della Città di Montepeloso (Oggi Irsina). Matera, Tip. F. P. Conti, 1901.

Matan, Ante. Životopis sv. Eufemije djevice i mučenice. Rovinj, Župa sv. Eufemije Rovinj, 2004.

Verronus, Paschalis. Vita diuae Euphemiae uirginis et martyris. Napoli, Carli e Pace, 1592.

\section{FONTI IN RETE:}

www.pedecerto.eu/public/index

www.pedecerto.eu/public/statistiche/speciale4_H_en.html?

www.pedecerto.eu/public/statistiche/schemi4_H_en.html? 
